\title{
Optimal Runge-Kutta Methods for First Order Pseudospectral Operators
}

\author{
J. L. Mead* and R. A. Renaut $\dagger$ \\ * College of Oceanic and Atmospheric Sciences, Oregon State University, Corvallis Oregon 97331-5503; \\ and $\dagger$ Department of Mathematics, Arizona State University, Tempe, Arizona 85287-1804 \\ E-mail: jmead@oce.orst.edu,renaut@asu.edu
}

Received November 17, 1998; revised March 12, 1999

\begin{abstract}
New Runge-Kutta methods for method of lines solution of systems of ordinary differential equations arising from discretizations of spatial derivatives in hyperbolic equations, by Chebyshev or modified Chebyshev methods, are introduced. These Runge-Kutta methods optimize the time step necessary for stable solutions, while holding dispersion and dissipation fixed. It is found that maximizing dispersion minimizes dissipation, and vice versa. Optimal methods with respect to large stability intervals on the imaginary axis and with respect to the eigenvalue spectra of the underlying pseudospectral discretizations are developed. In the latter case, stability regions are optimized to include the outliers of the spatial operators. Performance on a model problem in computational aeroacoustics is evaluated. The optimized schemes have two more function evaluations per timestep than the standard fourth order Runge-Kutta method, but allow timesteps up to 1.7 times larger. Moreover, dissipation and dispersion are reduced. (c) 1999 Academic Press

Key Words: Runge-Kutta; dissipation; dispersion; pseudospectral Chebyshev; hyperbolic equations; computational aeroacoustics.
\end{abstract}

\section{INTRODUCTION}

There has been much recent work on numerical methods for accurate and efficient computation of wave propagation [5-9, 16-21]. In [13], we analyze the effectiveness of the Chebyshev pseudospectral method (CPS) and a modified Chebyshev pseudospectral method (MPS) for the spatial discretization of the one-dimensional wave equation $u_{t}=u_{x}$. If CPS is used in space, then

$$
u_{t}=D u
$$


with

$$
D_{i j}=\left.\frac{d}{d x} T_{j}(x)\right|_{x=x_{i}},
$$

where $T_{j}(x)$ and $x_{i}$ are the Chebyshev polynomials and points, respectively [3]. The MPS method amounts to a preconditioning of the differential operator, $D$, by a diagonal matrix $A$, with entries dependent on the underlying spatial transformation. Here, and in [13], we consider the transformation introduced by Kosloff and Tal-Ezer [10] for which the entries of $A$ are given by

$$
A_{i i}=\frac{\sin ^{-1}(\alpha) \sqrt{1-\left(\alpha x_{i}\right)^{2}}}{\alpha},
$$

and for which we make the choice $\alpha=\cos (1 / N)$ in order to maximally damp the entries in $D$, see [13]. In both cases the matrices $D$ and $A D$ are dense. It is, therefore, imperative to design a solver in time which allows large timesteps while maintaining both stability and accuracy. Here we consider the design of optimal Runge-Kutta methods for the integration of systems with system matrix $D$ or $A D$. Equivalently, we require Runge-Kutta methods for which the stability regions enclose the scaled spectrum of the underlying system matrix. Thus, in Section 2, we review properties of the spectrum and pseudospectrum of the CPS and MPS derivative matrices, $D$ and $A D$, respectively.

The methods in $[6-9,16,17,20,21]$ are optimal in the sense that they require a small number of points per wavelength for minimal dispersion and dissipation. CPS and MPS require less than five points per wavelength for phase and amplitude errors $O\left(10^{-2}\right)[8,13]$. Thus, here we aim to improve the efficiency of the RK timestepping scheme, without introducing dissipation and dispersion, i.e., while maintaining the accuracy of the spatial operator. In Section 3 we show how to hold dispersion and dissipation fixed, while maximizing the stability region. In the process, we give the dissipation relations and show that maximizing the order of dispersion minimizes the order of dissipation, and vice versa, i.e., minimal dissipation and dispersion compete.

In Section 4 we derive two new RK methods of order 4 that allow time steps approximately 1.7 times larger than the usual fourth-order RK method. Finally, in Section 5 we evaluate the new RK methods in conjunction with CPS or MPS in space, for a benchmark problem in computational aeroacoustics [5].

\section{STABILITY}

The approach taken by Chiu and Kopriva in [4], for the determination of an optimal RK method, assumes that the eigenvalues of the CPS derivative operator dictate the size of the time step. When the system matrix is nonnormal, i.e., $D^{\mathrm{T}} D \neq D D^{\mathrm{T}}$, eigenvalue stability is not necessarily valid. Then the pseudospectrum determines the stability [14]: The pseudospectrum of a matrix $D$ is the set of eigenvalues of the matrix $D+E$, with $\|E\|<\epsilon$, i.e. all $\lambda \neq 0$ such that

$$
(D+E) x=\lambda x .
$$

It is well known, moreover, that the first order spatial derivative operator determined by the CPS method is non-normal [14]; thus the pseudospectrum is much larger than the 


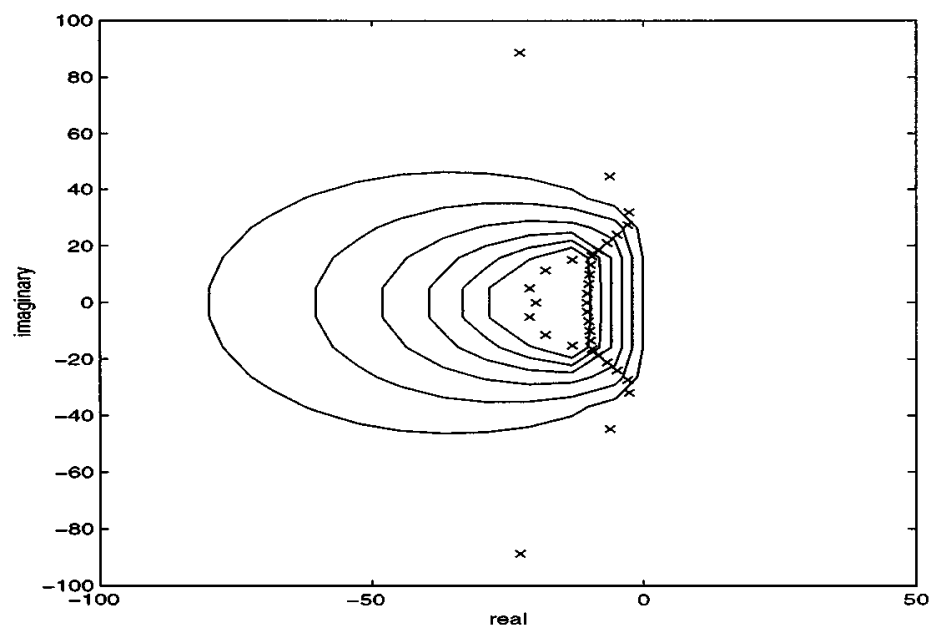

FIG. 1. Eigenvalues of CPS matrix $(x)$ and contours of its pseudo eigenvalues ( - ), for perturbations $10^{-6}, 10^{-5}, \ldots, 10^{-1}$. Outer contour is $10^{-1}$, and $N=32$.

spectrum. This is illustrated in Fig. 1 where we plot the spectrum and pseudospectrum for $\epsilon=10^{-6}, \ldots, 10^{-1}$. The largest contour refers to the boundary of the pseudospectrum when $\epsilon=10^{-1}$.

Figure 2 shows the same information for the MPS operator. The single contour reflects the pertubation $10^{-1}$; all other perturbations do not influence the spectrum. Note in each case that the outliers are insensitive to the perturbations.

Chiu and Kopriva were specifically interested to determine a method with fast convergence to steady state, rather than one that maintains high accuracy. Hence, in the development of their method they assumed large intervals of stability along the negative real axis and optimized to minimize dispersion. Consequently, their scheme is automatically dissipative and is not appropriate for accurate wave propagation for which the strength of the signal is important.

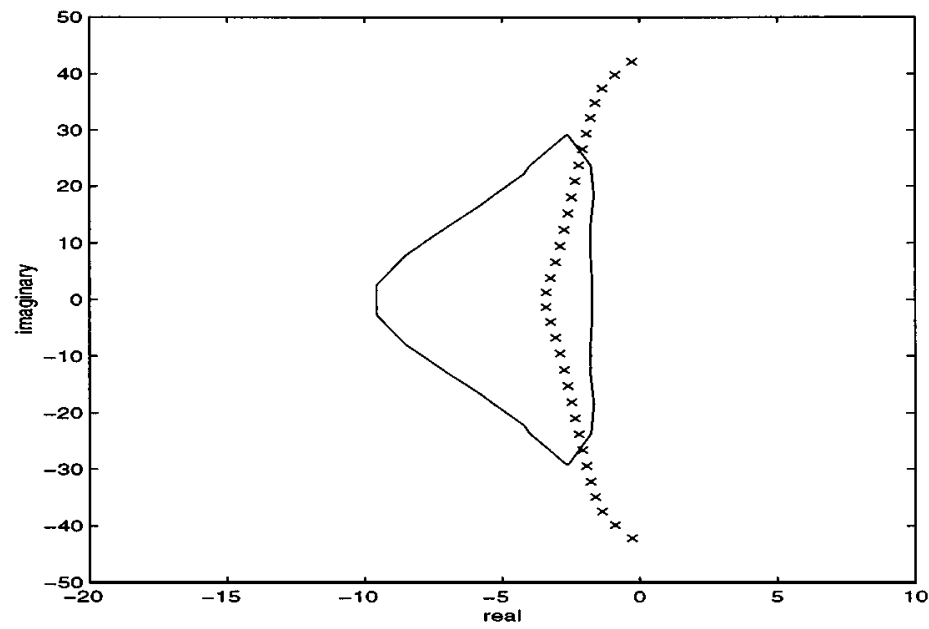

FIG. 2. Eigenvalues of MPS matrix $(\times)$. Single contour is pertubation $10^{-1}$; other pertubations do not effect the spectrum. $N=32$. 
We observe, from Fig. 1, that an optimal RK scheme for CPS would require both large negative real stability and a large interval of stability along the imaginary axis. These appear to be competing demands because, as we shall verify, a large interval of negative real stability introduces substantial dissipation, and is therefore not in general desirable. For the MPS method, however, we see that only large intervals along the imaginary axis are desirable. Hence we should be more successful in designing appropriate methods for the MPS operators. In Section 4 we describe an approach for finding the optimal schemes.

\section{DISPERSION AND DISSIPATION IN RUNGE-KUTTA METHODS}

Consider the solution of the initial value problem

$$
u_{t}=f(t, u)
$$

by the $s$-stage Runge-Kutta method

$$
\begin{aligned}
u_{n+1} & =u_{n}+h \sum_{i=1}^{s} b_{i} k_{i} \\
k_{i} & =f\left(t_{n}+c_{i} h, u_{n}+h \sum_{l=1}^{s} a_{i l} k_{l}\right) \\
c_{i} & =\sum_{j=1}^{s} a_{i j},
\end{aligned}
$$

where $b_{i}, c_{i}, a_{i j}$ are determined by the method. These coefficients are usually written in the Butcher array:

$$
\begin{array}{c|cccc}
c_{1} & a_{11} & a_{12} & \ldots & a_{1 s} \\
c_{2} & a_{21} & a_{22} & \ldots & a_{2 s} \\
\vdots & \vdots & & & \vdots \\
c_{s} & a_{s 1} & a_{s 2} & \ldots & a_{s s} \\
\hline b_{1} & b_{2} & \ldots & b_{s}
\end{array}
$$

or

$$
\frac{c \mid A}{\mid b^{\mathrm{T}}} .
$$

If $A$ is strictly lower triangular, the RK method is explicit. If $A$ is lower triangular the RK method is semi-implicit, and if $A$ is not lower triangular the RK method is implicit [11].

We follow the approach of Van Der Houwen and Sommeijer in [18] but emphasize the study of dissipation errors, rather than dispersion, or phase-lag errors. We consider the usual linear test equation,

$$
u_{t}=\lambda u, \quad \lambda=x+y i
$$

for which the exact solution is

$$
u(t+h)=e^{h(x+y i)} u(t)
$$


Using the notation of Albrecht [1], the RK solution has the form

$$
u_{n+1}=\left(1+h \lambda b^{\mathrm{T}} e+\cdots+(h \lambda)^{s} b^{\mathrm{T}} A^{s-1} e\right) u_{n},
$$

with $e=(1, \ldots, 1) \in \mathbf{R}^{s}$. If $\beta_{j}=b^{\mathrm{T}} A^{j-1} e$, then

$$
\begin{aligned}
u_{n+1} & =\left(1+h \lambda \beta_{1}+\cdots(h \lambda)^{s} \beta_{s}\right) u_{n} \\
& =\left(F_{s}+i G_{s}\right) u_{n}
\end{aligned}
$$

with

$$
\begin{aligned}
F_{s}= & 1+h x+\beta_{2} h^{2}\left(x^{2}-y^{2}\right)+\beta_{3} h^{3}\left(x^{3}-3 x y^{2}\right)+\beta_{4} h^{4}\left(x^{4}-6 x^{2} y^{2}+y^{4}\right) \\
& +\beta_{5} h^{5}\left(x^{5}-10 x^{3} y^{2}+5 x y^{4}\right)+\cdots+\beta_{s} h^{s}\left(x^{2}+y^{2}\right)^{s / 2} \cos s \theta \\
G_{s}= & h y+2 \beta_{2} h^{2} x y+\beta_{3} h^{3}\left(3 x^{2} y-y^{3}\right)+\beta_{4} h^{4}\left(4 x^{3} y-4 x y^{3}\right) \\
& +\beta_{5} h^{5}\left(5 x^{4} y-10 x^{2} y^{3}+y^{5}\right)+\cdots+\beta_{s} h^{s}\left(x^{2}+y^{2}\right)^{s / 2} \sin s \theta \\
\theta= & \tan ^{-1}(y / x) .
\end{aligned}
$$

DEFINITION 1 (Van Der Houwen and Sommeijer [18]). The RK method defined by (8) is dissipative of order $p$ if

$$
e^{x h}-\left|F_{s}+i G_{s}\right|=O\left(h^{p+1}\right)
$$

and dispersive of order $q$ if

$$
h y-\tan ^{-1}\left(G_{s} / F_{s}\right)=O\left(h^{q+1}\right) .
$$

When we expand all terms in Definition 1 up to order $p=5$, we see that a $p$ th order method is dissipative and dispersive of order $p$. In [18], Van Der Houwen and Sommeijer consider the case for which $x=0$, i.e., the exact solution is non-dissipative, and show that an order $p$ method has an order of dispersion of at least $2\lfloor(p+1) / 2\rfloor$.

In a 4th order method with $x=0, F_{s}$ and $G_{s}$ simplify to $F_{4}(h y)=1-\beta_{2}(h y)^{2}+\beta_{4}(h y)^{4}$ and $G_{4}(h y)=h y-\beta_{3}(h y)^{3}$. The dissipation error is

$$
D S(h y)=1-\left|F_{4}(h y)+i G_{4}(h y)\right|,
$$

and the dispersion error is

$$
D P(h y)=h y-\tan ^{-1}\left(G_{4}(h y) / F_{4}(h y)\right) .
$$

In Figs. 3 and 4 we plot the dissipation and dispersion errors versus $h y$ for a 2 nd $\left(\beta_{2}=\right.$ $\left.1 / 2, \beta_{3}=0, \beta_{4}=0\right), 3 \mathrm{rd}\left(\beta_{2}=1 / 2, \beta_{3}=1 / 6, \beta_{4}=0\right)$, and 4th $\left(\beta_{2}=1 / 2, \beta_{3}=\right.$ $\left.1 / 6, \beta_{4}=1 / 24\right)$ order method. The optimized method is described in Section 4.1. The dissipation error drops significantly from a 3 rd order to a 4 th order RK, i.e., from $O\left(10^{-5}\right)$ to $O\left(10^{-8}\right)$. Similarly, the dispersion error drops significantly from a 2 rd order to a 3 th order RK, i.e. from $O\left(10^{-4}\right)$ to $O\left(10^{-7}\right)$.

Van Der Houwen and Sommeijer determine the dispersion relations in [18]. In Table I we provide the corresponding dissipation relations. A detailed derivation of these dissipation relations and the expansions of the terms in Definition 1 is given in [13]. 

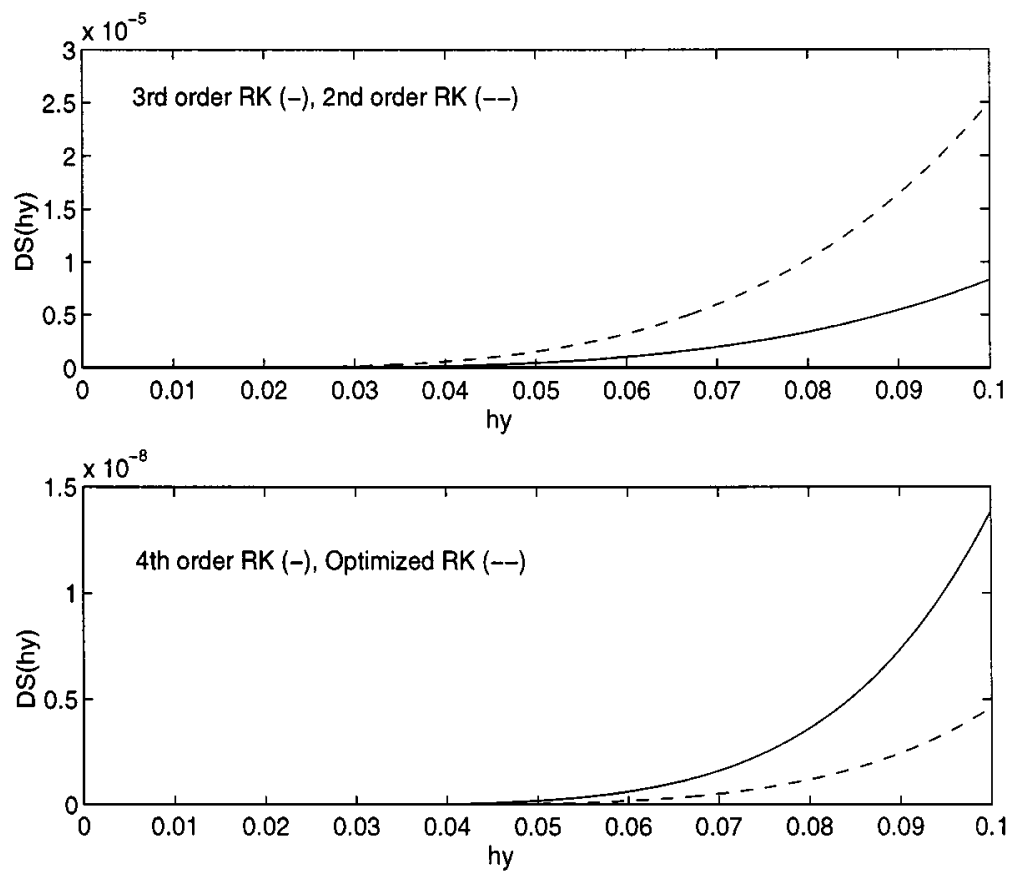

FIG. 3. Dissipation error (11) in a 2nd order, a 3rd order, a 4th order, and an optimized RK method.
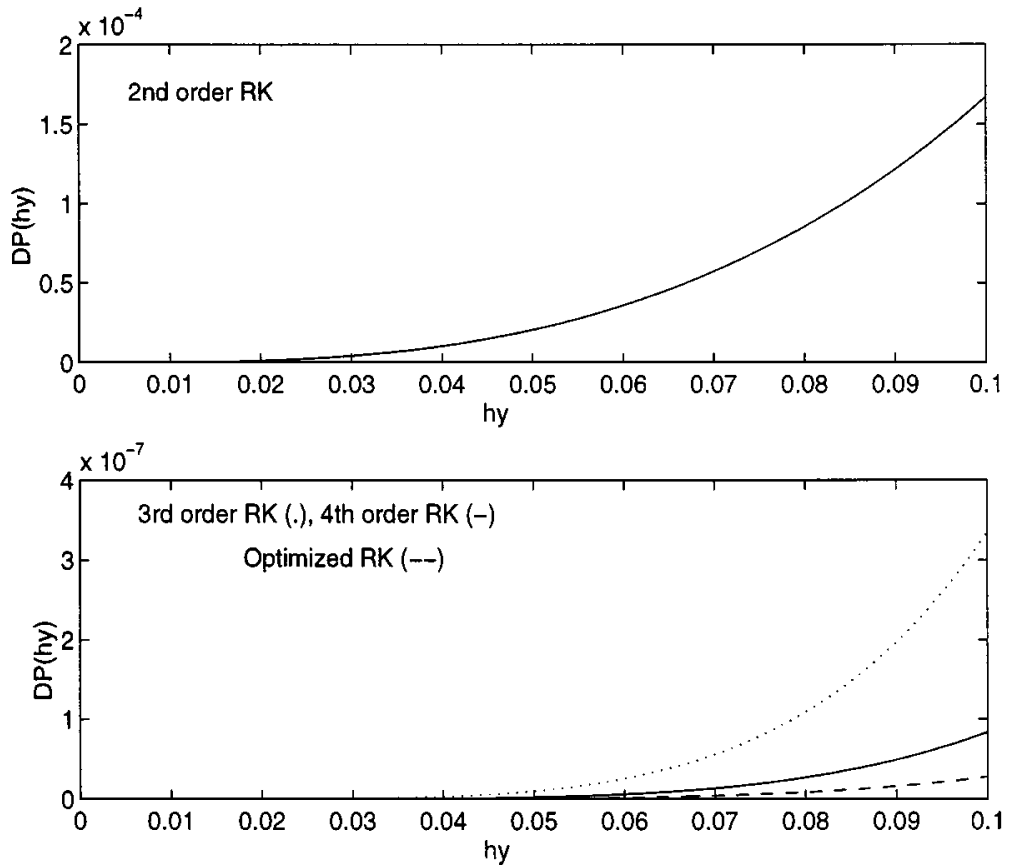

FIG. 4. Dispersion error (12) in a 2nd order, a 3rd order, a 4th order, and an optimized RK method. 


\section{TABLE I}

\section{Dissipation Relations}

\begin{tabular}{ccl}
\hline Order & Order of dissipation & \\
\hline 1,2 & 3 & $\beta_{2}=1 / 2$ \\
5 & $\beta_{3}-\beta_{4}=1 / 8$ \\
7 & $2 \beta_{5}-2 \beta_{6}+\beta_{3}^{2}-\beta_{4}=0$ \\
9 & $-2 \beta_{3} \beta_{5}-2 \beta_{7}+\beta_{4}^{2}+2 \beta_{8}+\beta_{6}=0$ \\
& & $\beta_{5}^{2}+2 \beta_{3} \beta_{7}-2 \beta_{10}+2 \beta_{9}-2 \beta_{4} \beta_{6}-\beta_{8}=0$ \\
& & $-2 \beta_{5} \beta_{7}+2 \beta_{12}+2 \beta_{4} \beta_{8}-2 \beta_{11}+\beta_{6}^{2}-2 \beta_{3} \beta_{9}+\beta_{10}=0$ \\
& $\beta_{2}=1 / 2, \beta_{3}=1 / 6, \beta_{4}=1 / 24$ \\
3,4 & $\beta_{5}-\beta_{6}=1 / 144$ \\
& & $\beta_{5}-6 \beta_{7}+1 / 192+6 \beta_{8}+3 \beta_{6}=0$ \\
& 7 & $12 \beta_{5}^{2}+4 \beta_{7}-24 \beta_{10}+24 \beta 9-\beta_{6}-12 \beta_{8}=0$ \\
9 & $-24 \beta_{5} \beta_{7}+2 \beta_{12}+\beta_{8}-24 \beta_{11}+12 \beta_{6}^{2}-4 \beta_{9}+12 \beta_{10}=0$ \\
& 11 &
\end{tabular}

Given the dispersion and dissipation relations, we can now determine the Runge-Kutta methods with a given number of stages which maximize the orders of dispersion and dissipation. The relations in Table I are simplified if we take

$$
\beta_{j}=0, \text { for } j>s \text {. }
$$

Thus, the coefficients that give the maximal order of dissipation can be determined by solving the resulting system. The number of stages, the maximum order of dissipation, and the required $\beta_{j}, j \leq s$, are given in Table II. The resulting order of dispersion is also listed.

TABLE II

Maximum Order of Dissipation

\begin{tabular}{|c|c|c|c|c|c|c|c|c|}
\hline $\begin{array}{l}\text { No. of } \\
\text { stages }\end{array}$ & $\begin{array}{l}\text { Order of } \\
\text { accuracy }\end{array}$ & $\begin{array}{c}\text { Order of } \\
\text { dissipation }\end{array}$ & $\begin{array}{c}\text { Order of } \\
\text { dispersion }\end{array}$ & $\beta_{2}$ & $\beta_{3}$ & $\beta_{4}$ & $\beta_{5}$ & $\beta_{6}$ \\
\hline 2 & 2 & 3 & 2 & $\frac{1}{2}$ & & & & \\
\hline 3 & 2 & 5 & 2 & $\frac{1}{2}$ & $\frac{1}{8}$ & & & \\
\hline 4 & 2 & 7 & 2 & $\frac{1}{2}$ & $\frac{1}{2} \pm \frac{1}{4} \sqrt{2}$ & $\frac{3}{8} \pm \frac{1}{4} \sqrt{2}$ & & \\
\hline 5 & 2 & 9 & 2 & $\frac{1}{2}$ & $\frac{1}{4}$ & $\frac{1}{8}$ & $\frac{1}{32}$ & \\
\hline 5 & 2 & 9 & 2 & $\frac{1}{2}$ & $\frac{-1+\sqrt{5}}{8}$ & $\frac{\sqrt{5}-2}{8}$ & $\frac{-11+5 \sqrt{5}}{64}$ & \\
\hline 5 & 4 & 7 & 4 & $\frac{1}{2}$ & $\frac{1}{6}$ & $\frac{1}{24}$ & $\frac{1}{144}$ & \\
\hline 6 & 2 & 11 & 2 & $\frac{1}{2}$ & $\frac{3}{16}$ & $\frac{1}{16}$ & $\frac{1}{64}$ & $\frac{1}{512}$ \\
\hline 6 & 2 & 11 & 2 & $\frac{1}{2}$ & $\frac{3+\sqrt{3}}{8}$ & $\frac{2+\sqrt{3}}{8}$ & $\frac{3 \sqrt{3}+5}{16(-1+\sqrt{3})}$ & $\frac{19+11 \sqrt{3}}{64(-1+\sqrt{3})}$ \\
\hline 6 & 2 & 11 & 2 & $\frac{1}{2}$ & $\frac{3-\sqrt{3}}{8}$ & $\frac{2-\sqrt{3}}{8}$ & $\frac{3 \sqrt{3}-5}{16(1+\sqrt{3})}$ & $\frac{-19+11 \sqrt{3}}{64(1+\sqrt{3})}$ \\
\hline 6 & 4 & 8 & 4 & $\frac{1}{2}$ & $\frac{1}{6}$ & $\frac{1}{24}$ & $\frac{1}{192}$ & $\frac{-1}{576}$ \\
\hline
\end{tabular}


TABLE III

Maximum Order of Dispersion

\begin{tabular}{ccccccccc}
\hline $\begin{array}{l}\text { No. of } \\
\text { stages }\end{array}$ & $\begin{array}{c}\text { Order of } \\
\text { accuracy }\end{array}$ & $\begin{array}{c}\text { Order of } \\
\text { dispersion }\end{array}$ & $\begin{array}{c}\text { Order of } \\
\text { dissipation }\end{array}$ & $\beta_{2}$ & $\beta_{3}$ & $\beta_{4}$ & $\beta_{5}$ & $\beta_{6}$ \\
\hline 2 & 1 & 4 & 1 & $1 / 3$ & & & & \\
2 & 2 & 4 & 3 & $1 / 2$ & & & & \\
3 & 1 & 6 & 1 & $2 / 5$ & $1 / 15$ & & & \\
3 & 3 & 4 & 3 & $1 / 2$ & $1 / 6$ & & & \\
4 & 1 & 8 & 1 & $3 / 7$ & $2 / 21$ & $1 / 105$ & & \\
4 & 3 & 6 & 3 & $1 / 2$ & $1 / 6$ & $1 / 30$ & & \\
4 & 4 & 6 & 5 & $1 / 2$ & $1 / 6$ & $1 / 24$ & & \\
5 & 1 & 10 & 1 & $4 / 9$ & $1 / 9$ & $1 / 63$ & $1 / 945$ & \\
5 & 3 & 8 & 3 & $1 / 2$ & $1 / 6$ & $4 / 105$ & $1 / 210$ & \\
5 & 5 & 6 & 5 & $1 / 2$ & $1 / 6$ & $1 / 24$ & $1 / 120$ & \\
6 & 1 & 12 & 1 & $5 / 11$ & $4 / 33$ & $2 / 99$ & $1 / 495$ & $1 / 840$ \\
6 & 3 & 10 & 3 & $1 / 2$ & $1 / 6$ & $-4 / 21$ & $-47 / 210$ & $-8 / 105$ \\
6 & 5 & 9 & 5 & $1 / 2$ & $1 / 6$ & $1 / 24$ & $1 / 120$ & \\
\hline
\end{tabular}

We see that a 6-stage method can have order of dissipation 11 , but the resulting order of dispersion is 2 .

The order of dispersion is maximized in a similar manner, using the dispersion relations provided in [18]. In Table III we summarize the number of stages, the maximum order of dispersion, the required $\beta_{j}$, and the resulting orders of dissipation. We see that it is possible for a 6-stage method to have order of dispersion 12, but the resulting order of dissipation is 1 . We conclude that the requirements of maximal order of dissipation and dispersion compete, a fact that the authors have not seen stated elsewhere.

\section{OPTIMAL STABILITY REGIONS}

The orders of dissipation and dispersion may be controlled by constraining the values of $\beta_{j}$, according to the dispersion and dissipation relations. It is thus possible to constrain the coefficients of $\beta_{j}$ to provide certain orders of dissipation and dispersion, while maintaining a limited number of degrees of freedom which can be used to optimize the scheme for the underlying spatial discretization. Note, however, that the stability regions of maximal order schemes, $p \leq 4$, cannot be maximized, because the number of stages is the same as the highest order, and there are no remaining degrees of freedom. If the order $p$ of the method is greater than 4, maximal order schemes constrain the coefficients $\beta_{1}, \ldots, \beta_{r}$ where $r<p$. Hence, the free parameters are $\beta_{j}, p<j<s$. For example, in [12], Lawson finds a value of $\beta_{6}$ that maximizes the real part of the stability region for the $p=5$ method. His method allows time steps twice as large as those for a fourth order method.

There is a tradeoff between the efficiency of the method and the maximal accuracy. As the order of accuracy increases, the number of function evaluations increases, and, hence, the cost increases. This is particularly significant when the underlying spatial operators are dense. We therefore choose a compromise and work with methods of 3 to 6 stages. Since higher accuracy leads to larger stability intervals, we only drop the maximum order of accuracy by one, in order to introduce free parameters. We require the following definition in the description of the algorithm. 
DEFINITION 2. The region of absolute stability is the set of all $h \lambda \in \mathbf{C}$ such that

$$
|r(h \lambda)| \leq 1, \quad \text { where } r(z)=\sum_{i=0}^{s} \beta_{i} z^{i}, \quad \beta_{0}=1 .
$$

Moreover the boundary of the stability region is given by the set of all $h \lambda \in \mathbf{C}$ such that

$$
|r(h \lambda)|=1
$$

\subsection{Extended Stability along the Imaginary Axis}

We noted in Section 2 that the pseudo-spectrum for the MPS operator extends along the imaginary axis without extension along the negative real axis. It is therefore appropriate to determine schemes with extended intervals of stability along the imaginary axis. Consequently, schemes designed with respect to large intervals of stability along the imaginary axis are useful for any differential operator with similar properties.

\section{ALGORITHM I.}

1. Hold $\beta_{1}, \ldots, \beta_{j}$ fixed for the desired order of dissipation and dispersion.

2. Maximize $f\left(\beta_{j+1}, \ldots, \beta_{s}\right)$ where $f$ is defined as follows:

2.1 Solve $r(z)=e^{i \theta_{l}}$ for a set of $\theta_{l}, 0 \leq \theta_{l}<2 \pi$ to provide roots $z_{k}\left(\theta_{l}\right)=x_{k l}+i y_{k l}$, $k=1,2, \ldots, s$ (see [2]).

$2.2 f\left(\beta_{j+1}, \ldots, \beta_{s}\right)=\max _{k, l}\left\{\left|y_{k l}\right|\right.$ such that $\left.x_{k l} \approx 0\right\}$.

\section{EXAMPLE.}

1. Let $s=2, j=1$ and $\beta_{1}=1$.

2.1 From (13) $r(z)=1+z+\beta_{2} z^{2}$, and assume $z_{k}=x_{k}+i y_{k}$ for $k=1,2$. The set of points $\left\{x_{k}\left(\theta_{l}\right), y_{k}\left(\theta_{l}\right)\right\}$ with $\theta_{l}=0, \ldots, 2 \pi$ that solve

$$
1+\left(x_{k}+i y_{k}\right)+\beta_{2}\left(x_{k}+i y_{k}\right)=e^{i \theta_{l}}
$$

define the stability boundary.

2.2 Consider first that (15) is solved numerically for $z_{k}\left(\theta_{l}\right)$. From the set of $z_{k}\left(\theta_{l}\right)$ with $x_{k}\left(\theta_{l}\right) \approx 0$,

$$
f\left(\beta_{2}\right)=\max _{k l}\left|y_{k}\left(\theta_{l}\right)\right|
$$

Alternatively, solve (15) exactly for $z_{k}\left(\theta_{l}\right)$. Let $x_{k}\left(\theta_{l}\right)=0$ for all $k, l$; then $y_{k}= \pm \sqrt{2 \beta_{2}-1} /$ $\beta_{2}$, and

$$
f\left(\beta_{2}\right)=\sqrt{2 \beta_{2}-1} / \beta_{2}
$$

In this case the maximum occurs when $\beta_{2}=1$.

Algorithm I was solved in Matlab, the function "roots" was used to solve $r(z)=e^{i \theta_{l}}$ for $z$, and "fmins" was used to maximuze $f\left(\beta_{j+1}, \ldots, \beta_{s}\right)$. The methods found are near optimal and results are shown in Table V. Vichnevetsky proves in [19] that the largest segment of the imaginary axis which is contained in the stability region cannot exceed $[-i(s-1), i(s-1)]$. 


\section{TABLE IV}

\section{Stability Intervals of Existing Methods}

\begin{tabular}{ccccc}
\hline Stages & $\begin{array}{c}\text { Order of } \\
\text { (accuracy, diss., disp.) }\end{array}$ & $\begin{array}{c}\text { Stability interval near imag. axis } \\
{[-\delta, \delta]}\end{array}$ & $\begin{array}{c}\text { Scaled interval } \\
{[-\delta / s, \delta / s]}\end{array}$ & \\
\hline 3 & $(3,3,4)$ & {$[-1.7871,1.7871]$} & {$[-.5957, .5957]$} & \\
4 & $(4,5,6)$ & {$[-2.8333,2.8333]$} & {$[-.7083, .7083]$} & \\
5 & $(4,7,4)$ & {$[-3.4642,3.4642]$} & {$[-.6928, .6928]$} & $\beta_{5}=1 / 144$ \\
6 & $(5,5,6)$ & {$[-4.1256,4.1256]$} & {$[-.6876, .6876]$} & $\beta_{6}=1 / 1280$ \\
\hline
\end{tabular}

Kinnmark and Gray [7] state the methods which obtain these upper bounds. These methods, however, are only second order accurate. We note that with Algorithm I for 3 stages we obtain their optimal method, $\beta_{1}=1, \beta_{2}=1 / 2, \beta_{3}=1 / 4$, as expected.

By comparing Table IV with Table V, we see that a six-stage fourth-order method almost doubles the imaginary stability interval of the four-stage fourth-order method (RK4). Thus, the time step for the optimized six-stage fourth-order method (RKM) can be almost twice that for RK4. RKM, however, requires two more function evaluations per step.

If RK4 requires a time step of size $h_{4}$ for stable solutions, there are $4 T / h_{4}$ function evaluations at the final time $T$. The stability intervals given in Tables IV and $\mathrm{V}$ indicate that RKM requires a time step of size $1.73 h_{4}$, and thus $3.47 T / h_{4}$ function evaluations at time $T$. This results in an effective step size increase of $15.26 \%$, which is significant for long time integrations.

The ability to almost double the time step with RKM was confirmed by plotting the stability region, and the scaled pseudo-spectra of the MPS derivative matrix; see Fig. 5. In the time integration, the spatial approximation is scaled by the time step $h$, so that it lies within the stability boundary.

In Table VI we give the largest time-step for RK4 and RKM with increasing $N$. These results confirm the approximate improvement of 1.7 to 1.8 for RKM over RK4.

\subsection{Extended Stability along the Imaginary Axis and the Negative Real Axis}

From Section 2 we observe that optimal RK methods to be used in conjunction with the CPS operator must extend both along the negative real axis and along the imaginary axis. In this case optimal methods were determined by applying Algorithm I, but with the

\section{TABLE V}

\section{Stability Intervals of Methods Found from Algorithm I}

\begin{tabular}{ccccc}
\hline Stages & $\begin{array}{c}\text { Order of } \\
(\text { accuracy, diss., disp. })\end{array}$ & $\begin{array}{c}\text { Stability interval near imag. axis } \\
{[-\delta, \delta]}\end{array}$ & $\begin{array}{c}\text { Scaled interval } \\
{[-\delta / s, \delta / s]}\end{array}$ & \\
\hline 3 & $(2,3,2)$ & {$[-2.0696,2.0696]$} & {$[-.6899, .6899]$} & $\beta_{3}=.25$ \\
4 & $(3,3,4)$ & {$[-2.8521,2.8521]$} & {$[-.7130, .7130]$} & $\beta_{4}=.03812$ \\
5 & $(3,3,4)$ & {$[-3.9356,3.9356]$} & {$[-.7871, .7871]$} & $\beta_{4}=.03255$ \\
& & & & $\beta_{5}=.00633$ \\
6 & $(4,5,4)$ & {$[-4.8984,4.8984]$} & {$[-.8164, .8164]$} & $\beta_{5}=.00556$ \\
& & & & $\beta_{6}=.00093$ \\
\hline
\end{tabular}



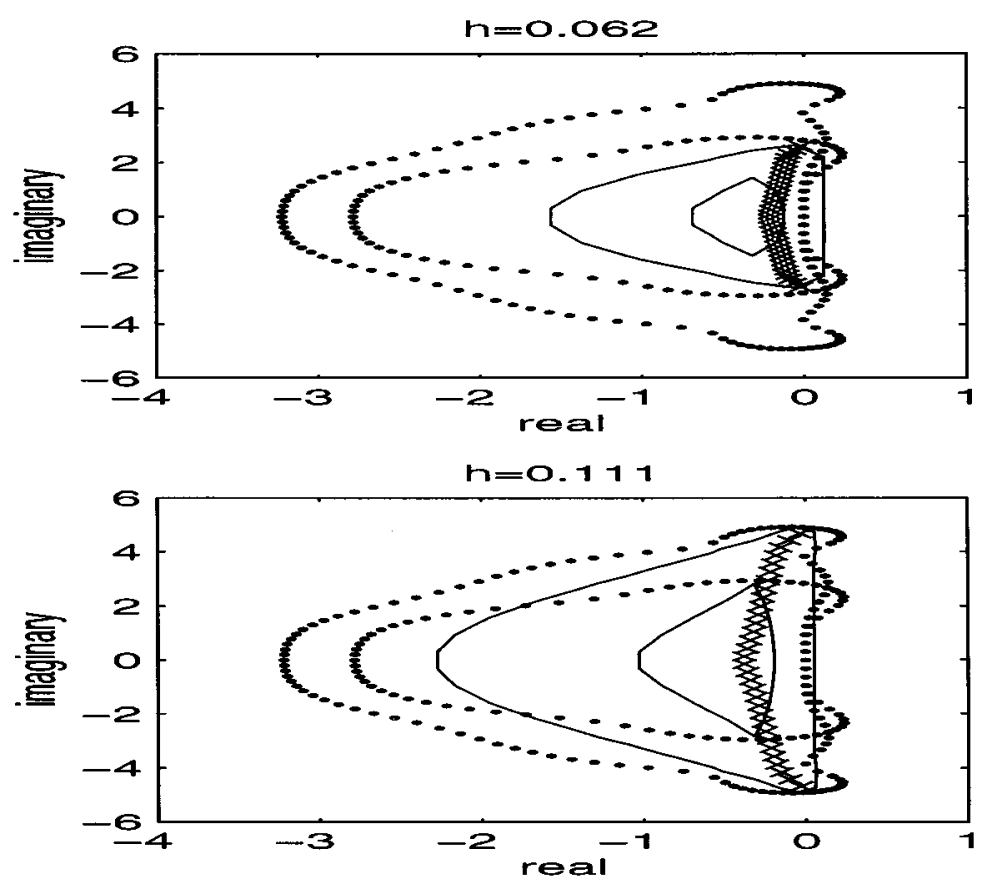

FIG. 5. Eigenvalues $(\times)$ and pseudo spectra ( - ) of MPS matrix, $N=32$. Stability regions for RKM (outer .), and RK4 (inner .).

additional constraints that the outlier eigenvalues for $N=32$, which are insensitive with regard to perturbations, lie inside the stability region.

Algorithm II. This is the same as Algorithm I, but do not require $x_{k l} \approx 0$, i.e., replace 2.2 by

$$
\text { 2.2' } f\left(\beta_{j+1}, \ldots, \beta_{s}\right)=\max _{k j}\left\{\left|y_{k j}\right|\right\} .
$$

The six-stage fourth-order method (RKC) defined by

$$
\beta_{6}=6.42853125 e-04 \quad \beta_{5}=.005676975
$$

was found from Algorithm II. It has a stability region which reaches out to include the outlying eigenvalues of the CPS derivative matrix; see Fig. 6.

\section{TABLE VI}

The Largest Allowable Timesteps Which Ensure That the Pseudospectra of MPS Lie within the Stability Region of RK4 or RKM

\begin{tabular}{rrrrr}
\hline \multirow{2}{*}{$N$} & \multicolumn{2}{c}{$h$} & & Ratio \\
\cline { 2 - 4 } & RK4 & RKM & $\begin{array}{c}\text { \% increase } \\
\text { in efficiency }\end{array}$ \\
\hline 16 & 0.1440 & 0.2470 & 1.72 & 14.35 \\
32 & 0.0620 & 0.1110 & 1.80 & 19.35 \\
64 & 0.0300 & 0.0520 & 1.73 & 15.56 \\
100 & 0.0180 & 0.0325 & 1.81 & 20.37 \\
128 & 0.0143 & 0.0251 & 1.76 & 17.02 \\
\hline
\end{tabular}




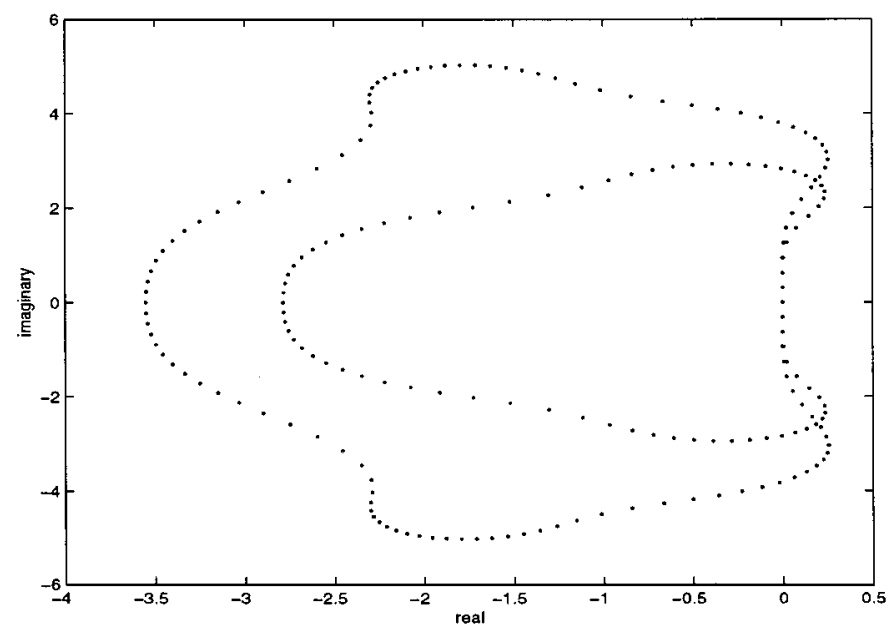

FIG. 6. Stability boundary of RKC (outer .) and RK4 (inner .).

Again, the ability to increase the time step was confirmed by plotting the scaled pseudospectrum of the CPS matrix with the regions of stability of RKC and RK4 in Fig. 7. The scaled pseudo spectrum lies entirely within the stability regions of RKC or RK4, for $\epsilon<10^{-2}$. Table VII shows that the increased time step with RKC, as compared to RK4, is largely independent of $N$.

The values of the RKM and RKC coefficients are not unique, but the methods must be 4 th order, i.e., from [1],

$$
\begin{gathered}
b^{\mathrm{T}} e=1 \quad b^{\mathrm{T}} c=\frac{1}{2} \quad b^{\mathrm{T}} c^{2}=\frac{1}{3} \\
b^{\mathrm{T}} c^{3}=\frac{1}{4} \quad b^{\mathrm{T}} A c=\frac{1}{6} \quad b^{\mathrm{T}} A c^{2}=\frac{1}{12} \\
b^{\mathrm{T}} A^{2} c=\frac{1}{24} \quad \frac{1}{2} b^{\mathrm{T}} D c^{2}=b^{\mathrm{T}} D A c
\end{gathered}
$$

$D=\operatorname{diag}\left(c_{i}\right)$. In addition, both RKM and RKC have the condition that

$$
\beta_{6}=k_{1}, \quad \beta_{5}=k_{2},
$$

for the $k_{1}, k_{2}$ found in the optimization process. This nonlinear system of 10 equations and

TABLE VII

The Largest Allowable Time Steps Which Ensure the Pseudospectra of CPS Lie within the Stability Region of RK4 or RKC

\begin{tabular}{rlcrr}
\hline \multirow{2}{*}{$N$} & \multicolumn{2}{c}{$h$} & & \% increase \\
\cline { 2 - 3 } & RK4 & RKC & Ratio & in efficiency \\
\hline 16 & 0.1240 & 0.1980 & 1.60 & 6.45 \\
32 & 0.0310 & 0.0520 & 1.68 & 11.83 \\
64 & 0.0079 & 0.0132 & 1.67 & 11.39 \\
100 & 0.0032 & 0.0055 & 1.72 & 14.58 \\
128 & 0.0020 & 0.0034 & 1.70 & 13.33 \\
\hline
\end{tabular}



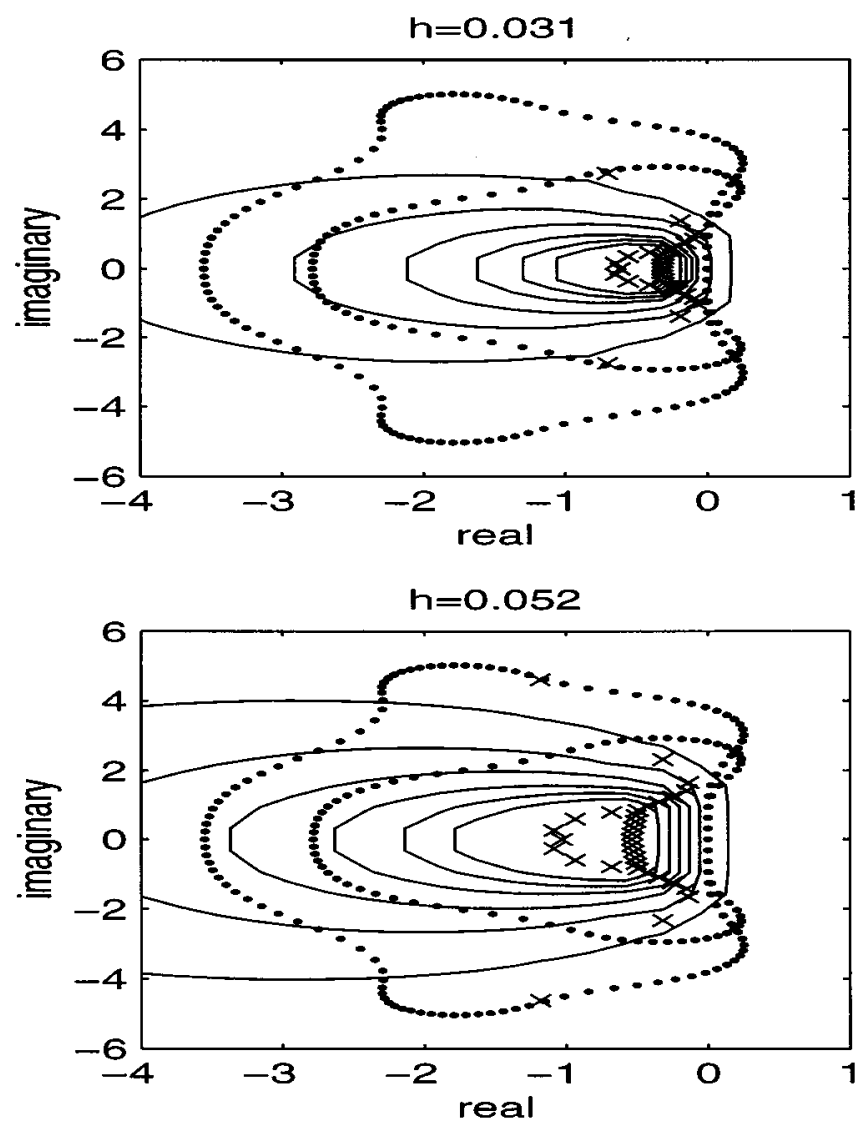

FIG. 7. Eigenvalues $(\times)$ and pseudo spectra (-) of CPS matrix, $N=32$. Stability regions for RKC (outer .), and RK4 (inner .).

21 unknowns can be reduced to 10 equations and 10 unknowns if we assume the method is of the form

$$
\begin{array}{c|cccccc}
0 & 0 & & & & & \\
c_{2} & c_{2} & & & & & \\
c_{3} & 0 & c_{3} & & & & \\
c_{4} & 0 & 0 & c_{4} & & & \\
c_{5} & 0 & 0 & 0 & c_{5} & & \\
c_{6} & 0 & 0 & 0 & 0 & c_{6} & \\
\hline & b_{1} & b_{2} & b_{3} & b_{4} & 0 & b_{6}
\end{array} .
$$

The values of the RK coefficients are given by

$$
\begin{array}{lll}
b_{1}=-0.15108370762927 & b_{2}=0.75384683913851 & b_{3}=-0.36016595357907 \\
b_{4}=0.52696773139913 & b_{5}=0 & b_{6}=0.23043509067071 \\
c_{2}=0.16791846623918 & c_{3}=0.48298439719700 & c_{4}=0.70546072965982 \\
c_{5}=0.09295870406537 & c_{6}=0.76210081248836, &
\end{array}
$$


and

$$
\begin{array}{lll}
b_{1}=-1.11863930033618 & b_{2}=2.50614037113582 & b_{3}=-2.22307558659639 \\
b_{4}=0.99978067105009 & b_{5}=0 & b_{6}=0.83579384474665 \\
c_{2}=0.11323867464627 & c_{3}=0.38673801369281 & c_{4}=0.62314978336040 \\
c_{5}=0.05095678842127 & c_{6}=0.54193120548949 . &
\end{array}
$$

for RKM and RKC, respectively.

\section{SOLUTION OF SPHERICAL WAVE PROBLEM}

To evaluate the effectiveness of the optimized RK methods a benchmark problem in computational aeroacoustics [5] was solved:

$$
\begin{array}{cl}
\frac{\partial u}{\partial t}+\frac{\partial u}{\partial r}+\frac{u}{r}=0 & 5 \leq r \leq 315, t>0 \\
u(r, 0)=0 & 5 \leq r \leq 315 \\
u(5, t)=\sin (\pi t / 3) & 0<t<300 .
\end{array}
$$

The analytic solution is

$$
u(r, t)= \begin{cases}0, & r>t+5 \\ 5[\sin (\pi(t-r+5) / 3)] / r, & r \leq t+5\end{cases}
$$

Figure 8 illustrates the analytic solution on the entire domain. Errors in the numerical solution will be seen on the right side of the graph, where the wave dies out. Thus, as in [5], the numerical results are plotted on the domain $250 \leq r \leq 330$.

Figures 9 and 10 illustrate the solutions for $N=270$, for which there are five points per wavelength; details are provided in [13]. The time steps are chosen so that the absolute error measured in the maximum norm is $O\left(10^{-3}\right)$. In Fig. 9, the graph of the solution found with RK4 is identical (up to order $10^{-3}$ ) to the graph of the solution found with RKC. Correspondingly in Fig. 10, the RK4 solution is identical to the RKM solution.

Execution times, provided in Table VIII, are for a Fortran code running on a dedicated HP 9000/735 system. We conclude that RKM provides the optimal algorithm while maintaining accuracy equivalent to that of the CPS operator.

\section{TABLE VIII}

Summary of Methods and Their Execution Times

\begin{tabular}{cclcc}
\hline Spatial approx. & Temporal approx. & Step size & Error & Time \\
\hline CPS & RK4 & 0.0004 & $3 e-3$ & $32 \mathrm{~h} 6$ min $39 \mathrm{~s}$ \\
CPS & RKC & 0.00067 & $3 e-3$ & $28 \mathrm{~h} 32 \min 5 \mathrm{~s}$ \\
MPS & RK4 & 0.1 & $2 e-3$ & 8 min $14 \mathrm{~s}$ \\
MPS & RKM & 0.2 & $2 e-3$ & 6 min $2 \mathrm{~s}$ \\
\hline
\end{tabular}




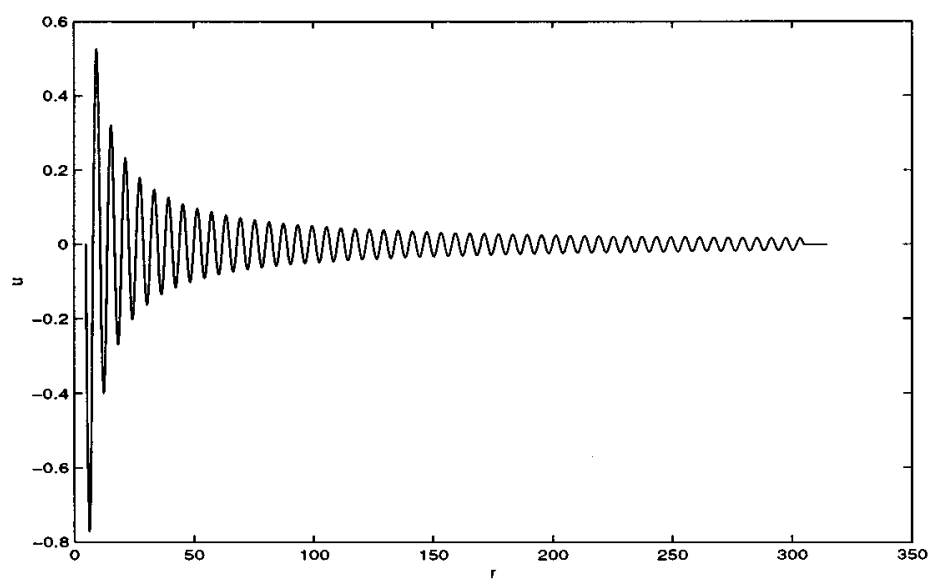

FIG. 8. True solution of benchmark problem at $t=300$.
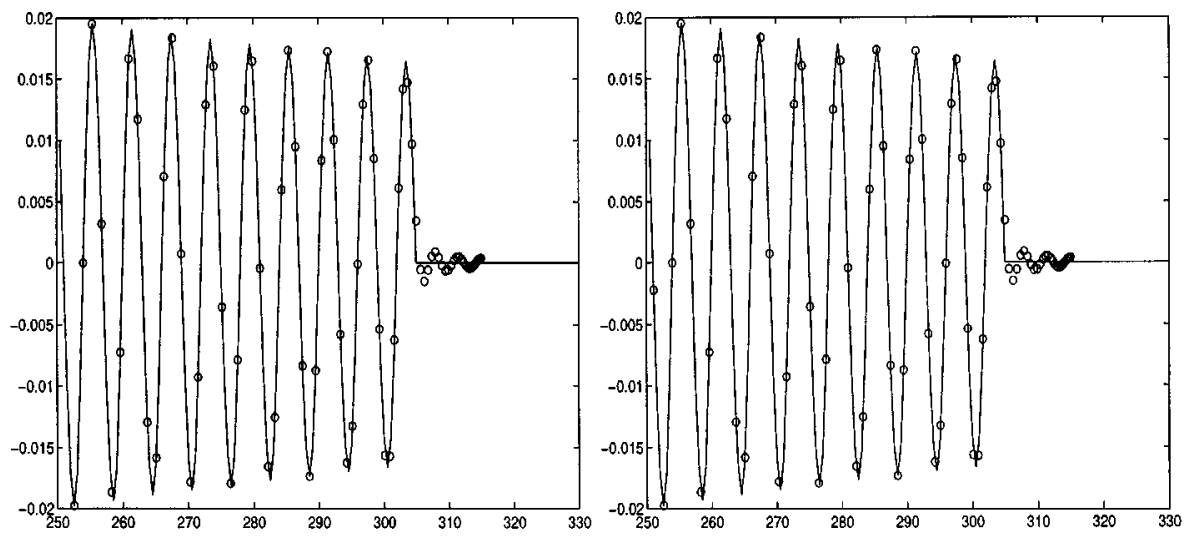

FIG. 9. Solution of benchmark problem with CPS: the true solution (-) and the computed solution (o). RK4 time $\approx 32 \mathrm{~h}$ (left), RKC time $\approx 28 \mathrm{~h}$ (right).
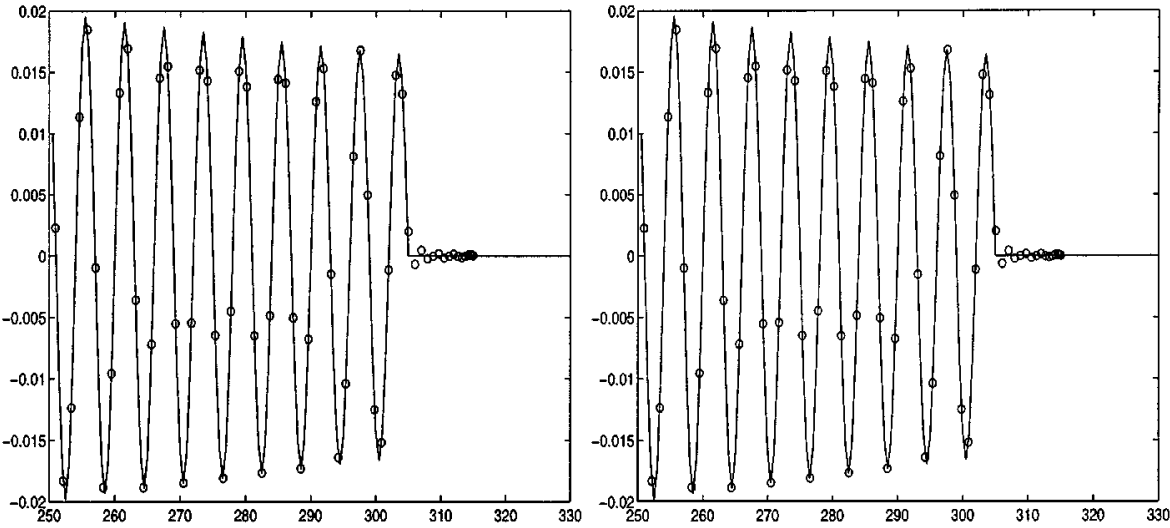

FIG. 10. Solution of benchmark problem with MPS: the true solution (-) and the computed solution (o). RK4 time $\approx 8 \mathrm{~min}$ (left); RKM time $\approx 6 \mathrm{~min}$ (right). 


\section{ACKNOWLEDGMENTS}

The work of the first author was supported by the NASA Space Grant Program. The second author was supported under NSF Grant DMS-9402943.

\section{REFERENCES}

1. P. Albrecht, The Runge-Kutta theory in a nutshell, SIAM J. Numer. Anal. 33(5), 1712 (1996).

2. J. C. Butcher, The Numerical Analysis of Ordinary Differential Equations: Runge-Kutta and General Linear Methods (Wiley, New York, 1987).

3. C. Canuto, M. Y. Hussaini, A. Quarteroni, and T. A. Zang, Spectral Methods in Fluid Dynamics (SpringerVerlag, Berlin/New York, 1988).

4. C. Chiu and D. A. Kopriva, An optimal Runge-Kutta method for steady-state solutions of hyperbolic systems, SIAM J. Numer. Anal. 29(2), 425 (1992).

5. J. C. Hardin, J. R. Ristorcelli, and C. K. W. Tam (editors), ICASE/LaRC Workshop on Benchmark Problems in Computational Aeroacoustics, presented at NASA CP 3300 (1995).

6. F. Q. Hu, M. Y. Hussaini, and J. Mathey, Low-Dissipation and -Dispersion Runge-Kutta Schemes for Computational Acoustics, ICASE Report 94-102 (1994).

7. I. P. E. Kinnmark and W. G. Gray, One step integration methods with maximum stability regions, Math. Comput. Simulation 26, 181 (1984).

8. D. A. Kopriva, Spectral Solution of Acoustic Wave Propagation Problems, presented at AIAA 13th Aeroacoustics Conference (1990).

9. D. A. Kopriva and J. H. Kolias, Solution of Acoustic Workshop Problems by a Spectral Multidomain Method, presented at NASA CP 3300 (1995).

10. D. Kosloff and J. Tal-Ezer, A modified Chebyshev pseudospectral method with an $O\left(N^{-1}\right)$ time step restriction, J. Comput. Phys. 104, 457 (1993).

11. J. D. Lambert, Numerical Methods for Ordinary Differential Systems (Wiley, New York, 1991).

12. J. D. Lawson, An order five Runge-Kutta process with extended region of stability, SIAM J. Numer. Anal. 3(4), 593 (1966).

13. J. L. Mead, Numerical Methods for Problems in Computational Aeroacoustics, Ph.D. dissertation, Arizona State University, 1998.

14. S. C. Reddy and L. N. Trefethen, Stability of the method of lines, Numer. Math. 62, 235 (1992).

15. S. C. Reddy, J. A. C. Weideman, and G. F. Norris, On a Modified Chebyshev Pseudospectral Method (unpublished, Oregon State University, Corvalis, OR).

16. C. K. W. Tam and J. C. Webb, Dispersion-relation-preserving finite difference schemes for computational acoustics, J. Comput. Phys. 107, 262 (1993).

17. C. K. W. Tam, Computational aeroacoustics: Issues and methods, AIAA J. 33(10), 1788 (1995).

18. P. J. Van Der Houwen and B. P. Sommeijer, Explicit Runge-Kutta (-Nyström) methods with reduced phase errors for computing oscillating solutions, SIAM J. Numer. Anal. 24, 595 (1987).

19. R. Vichnevetsky, New stability theorems concerning one-step numerical methods for ordinary differential equations, Math. Comput. Simulation 25, 199 (1983).

20. D. W. Zingg, H. Lomax, and H. Jurgens, High-accuracy finite-difference schemes for linear wave propagation, SIAM J. Sci. Comput. 17(2), 328 (1996).

21. D. W. Zingg, A review of high-order and optimized finite-difference methods for simulating linear wave phenomena, AIAA Paper 97-2088, in Proceedings of the 13th AIAA Computational Fluid Dynamics Conf. (1996). 